\title{
EL CONSUMO DE SUSTANCIAS COMO FACTOR DE RIESGO \\ PARA LA CONDUCTA DELICTIVA: UNA REVISIÓN SISTEMÁTICA
}

\author{
SUBSTANCE ABUSE AS A RISK FACTOR FOR \\ CRIMINAL BEHAVIOR: \\ A SYSTEMATIC REVIEW
}

ESTHER PÉREZ ${ }^{1}$ Y SERGIO RUIZ ${ }^{1}$

Cómo referenciar este artículo/How to reference this article:

Pérez, E. y Ruiz, S. (2017). El consumo de sustancias como factor de riesgo para la conducta delictiva: Una revisión sistemática [Substance Abuse as a Risk Factor for Criminal Behavior: A Systematic Review]. Acción Psicológica, 14(2), 33-50. https://doi.org/10.5944/ap.14.2.20748

\section{Resumen}

La delincuencia es un problema social que merece el estudio de sus causas para su prevención. A lo largo de la historia, se ha estudiado la asociación entre el consumo de sustancias y el crimen, aunque esta relación no está clara. Este trabajo tiene como objetivo conocer los últimos hallazgos relativos a la consideración del consumo como factor de riesgo para el delito. Se trata de una Revisión Sistemática de trabajos empíricos concernientes al estudio del consumo como posible factor de riesgo de desarrollo de conductas delictivas, publicados entre 2010 y 2015 , en español, inglés y portugués. Se realizó una búsqueda sistemática en Scopus, PsycINFO, Medline y Psicodoc, se incluyeron treinta y dos estudios que se revisaron con una lista de comprobación de calidad de artículos epidemiológicos. Como resultados se indica que la mayoría de los trabajos incluidos se centraban en el consumo de alcohol, de cannabis o el policonsumo, y casi un $80 \%$ coinciden en que el consumo representa un factor de riesgo en el desarrollo de ciertas tipologías delictivas. Como conclusión, conocer este factor de riesgo, podría utilizarse como medio de prevención. Finalmente, se destaca la posibilidad de sesgo de información.

Palabras clave: Delincuencia; Consumo de sustancias; Factores de riesgo; Revisión sistemática.

Correspondencia: Esther Pérez. Departamento de Psicología Social, Facultad de Psicología. Universidad de Granada.

Email: estherperezca@,correo.ugr.es

ORCID: Esther Pérez (https://orcid.org/0000-0003-0524-4904) y Sergio Ruiz (http://orcid.org/0000-0002-4244-2777)

${ }^{1}$ Universidad de Granada, España.

Recibido: 17 septiembre de 2017.

Aceptado: 09 de noviembre de 2017. 


\begin{abstract}
Delinquency is a social problem that deserves study to be prevented. Throughout history, the association between substance abuse and crime has been studied, although this relationship is unclear. The main aim of this paper is to present the latest findings concerning the consideration of substance use as a risk factor for crime. This paper is a systematic review of empirical studies regarding the study of substance as a possible risk factor for the development of criminal conduct, published between 2010 and 2015, in Spanish, English and Portuguese. A systematic search at Scopus, PsycINFO, Medline and Psicodoc was carried out, including a total of thirty-two studies reviewed with a checklist of the quality of epidemiological papers. The majority of papers focused on alcohol, cannabis or poly-drug use, and almost $80 \%$ agree that consumption is a risk factor for the development of criminal behavior and some types of crimes. This review concludes that the knowledge that consumption represents a risk factor could be used as a means of prevention. The possibility of information bias limitation is highlighted.
\end{abstract}

Keywords: Delinquency; Substance use; Risk factors; Systematic review.

\section{Introducción}

A lo largo del tiempo, las investigaciones han relacionado el consumo de sustancias con las conductas delictivas, pero ¿Es el consumo realmente un factor de riesgo? ¿Tiene algún papel en la aparición de estas conductas? Si revisamos las investigaciones que pretenden establecer la etiología del delito, comúnmente se han centrado en establecer una serie de variables asociadas a este tipo de conducta como, por ejemplo, las variables relativas al individuo, socioeconómicas y psicosociales.

Algunos estudios como el de Montgomery, Thomson y Barczyk (2011), indicaron que el $40 \%$ de la varianza del delito podía explicarse únicamente por variables individuales (como el consumo de sustancias y algunas psicopatologías). Si nos centramos en la variable del consumo y/o abuso de sustancias, un buen número de traba- jos recientes coinciden en que se puede asociar al desarrollo de conductas delictivas (Gatti, Soellner, Schadee, Verde y Rocca, 2013; Miller et al., 2016; Nordstrom y Dackis, 2011). Sin embargo, la dirección de la asociación entre las variables no queda clara, y aún no se conoce si las drogas operan realmente como un factor de riesgo o predictor (Swahn y Donovan, 2004), un factor mediador o asociado, o bien una consecuencia de la actividad delictiva. De aquí surge la necesidad de revisar y estudiar aquellos trabajos cuyo diseño y análisis estadístico se centre en comprobar si el consumo de sustancias supone realmente un riesgo en la aparición de conducta delictiva, sabiendo que, como veremos a continuación, los datos del consumo de drogas son como mínimo preocupantes.

En Europa, uno de cada cuatro ciudadanos ha consumido drogas ilícitas y, actualmente, el consumo engloba una gran cantidad de sustancias en comparación con el pasado, aunque la sustancia ilegal más prevalente es el cannabis (Observatorio Europeo de las Drogas y las Toxicomanías [EMCDDA], 2016). En cuanto al alcohol, los datos proporcionados por la Organización Mundial de la Salud (OMS, 2014) indican que en el año 2010 el consumo mundial de alcohol fue de más de seis litros por persona. Es de destacar también, algunos datos relativos al consumo de alcohol y sustancias ilícitas entre los jóvenes españoles, ya que, por ejemplo, la edad media del inicio del consumo de alcohol bajó de diecinueve años aproximadamente en 2006, a diecisiete años en 2013 (Ministerio de Sanidad, Servicios Sociales e Igualdad, 2013). Las drogas de consumo más prevalente en España son, en orden: alcohol, tabaco, hipnosedantes, cannabis y cocaína (Observatorio Español de la Droga y las Toxicomanías [OEDT], 2015). Esta misma fuente establece que la edad media del inicio del consumo de sustancias psicoactivas como el cannabis, está en torno a los diecinueve años, indicando además que es la droga más prevalente después del alcohol entre los más jóvenes. No se ha de olvidar los fármacos y otras sustancias, cuyo consumo también se ha relacionado con el desarrollo de conductas violentas (Ford, 2008; Pope, Kouri y Hudson, 2000).

En cuanto al delito, aunque las estadísticas europeas indican que la tendencia de la delincuencia es a la disminución (Oficina Europea de Estadística [EUROSTAT], 
2010), los datos estadísticos en España indican más de 59970 ciudadanos son reclusos, de los cuales más de 4180 tienen entre dieciocho y veinticinco años (Secretaría General de Instituciones Penitenciarias, 2016) y que el número de menores que delinquen es todavía mayor, 13981 menores de entre catorce y diecisiete años (Instituto Nacional de Estadística [INE], 2015).

Los datos proporcionados del delito, y los datos en relación al consumo de sustancias, además de la asociación confusa entre estas variables, establecida a lo largo del estudio de las causas del delito, nos inclinaron a centrarnos y tratar de conocer mejor esta posible relación de riesgo. Por tanto, el propósito que se planteó para la revisión fue conocer los resultados de los últimos trabajos que estudian la influencia que ejerce la variable de consumo de sustancias en el desarrollo de conductas delictivas, y por tanto confirmar si es realmente un factor de riesgo en la aparición del delito. El conocimiento de la dirección de esta influencia y la síntesis de los resultados de estudios empíricos de los últimos cinco años, podría ser relevante para el desarrollo de programas de prevención de la delincuencia por la posible participación del consumo de sustancias en su etiología.

\section{Método}

\section{Procedimiento}

Se realizó una búsqueda bibliográfica y sistemática de documentos en cuatro bases de datos: Scopus, Medline, Psicodoc y PsycINFO (en ese orden). La búsqueda y selección de artículos comenzó el 11 de Julio y finalizó el 27 de Julio de 2016. El procedimiento de selección de los artículos siguió una estrategia jerárquica consistente en primer lugar en seleccionar los trabajos en función de la lectura de su título, resumen y palabras clave, para comprobar si seguían las directrices marcadas por los criterios de inclusión y exclusión establecidos (consultar en los apartados de "Criterios de inclusión" y "Criterios de exclusión").

Los términos de búsqueda utilizados se adaptaron al idioma que admitía cada base de datos y se procedió a refinar los resultados según: periodo de tiempo, idioma y tipo de documento ("artículos" y "revisiones"). Los términos utilizados fueron los siguientes:

«predictors OR epidemiology OR "risk factors" AND delinquency OR crime OR "criminal behavior" OR offense OR felony OR crim * OR delinq * AND drugs OR "substance abuse" OR "drug use" OR "substance use" OR alcohol OR cocaine OR cannabis OR heroin OR drug* AND "case control” OR cohort OR prospective OR longitudinal».

En segundo lugar, se procedió a leer los documentos completos y se revisó su calidad mediante la declaración STROBE -Strengthening the Reporting of Observational studies in Epidemiology- (Von Elm et al., 2008). Se trata de una lista de puntos a tener en cuenta en la publicación de estudios observacionales epidemiológicos, constituyendo una guía de recomendaciones para comunicar y proceder en este tipo de estudios. La lista contiene veintidós puntos esenciales relacionados con aspectos en la redacción de los artículos y el procedimiento del estudio que deben presentar o tener en cuenta estos estudios. Los artículos que no cumplían más de dos criterios de la lista se descartaron y no se incluyeron en esta revisión. Aquellos que no cumplían dos puntos pertenecientes al mismo apartado de la lista de comprobación, se descartaron también.

\section{Griterios de inclusión}

Los criterios de inclusión para la selección de los artículos y en función de los objetivos planteados, consistieron en: estudios empíricos observacionales y/o cuasi- experimentales publicados entre 2010 y 2015; que evalúen posibles relaciones causales entre el consumo de drogas, sustancias o alcohol, con conductas delictivas, violentas o criminales; con un diseño que permita la estimación de este factor como un factor de riesgo o de riesgo relativo (diseño longitudinal, caso-control, retrospectivo, prospectivo, o de cohortes, análisis odds ratio, análisis del riesgo relativo); que hayan sido publicados en Inglés, Español o Portugués; que cuenten con muestras de población que haya cometido delitos y/o consumido drogas en el presente o en el pasado; y cuyos datos recogidos tengan una procedencia tanto oficial como autoin- 
formada, puesto que trabajos como el de Dubow, Huesmann, Boxer y Smith (2014) señalan que hay una correspondencia de moderada a alta entre los datos recogidos mediante autoinformes y los datos oficiales.

\section{Criterios de exclusión}

Se descartaron de esta revisión: estudios descriptivos; estudios relacionados con el concepto de reincidencia; enfocados en una población muy específica; que no cumpliesen los criterios de calidad o con diseños y/o análisis de datos no encaminados a estimar el riesgo relativo, vulnerabilidad o causalidad, basándonos en las ideas y métodos que exponen Pita, Vila y Carpente (1997) relativas a la estimación del riesgo. Según estos autores para determinar factores de riesgo se debe calcular o bien el riesgo absoluto o bien el riesgo relativo, que se estima calculando el Odds Ratio, y que mide la fuerza de asociación entre la exposición a un factor y su consecuencia.

\section{Resultados}

\section{Estudios seleccionados}

Siguiendo el procedimiento ya descrito, se incluye de forma esquemática el procedimiento de descarte y selección de estudios en la Figura 1. De los incluidos, veinte procedían de Scopus, ocho de Medline y cuatro de PsycINFO. La información extraída de los estudios se organizó utilizando una tabla que aparece a continuación, en la que se detallan las características de la muestra, el tipo de análisis y los principales resultados de cada estudio (Apéndice A).

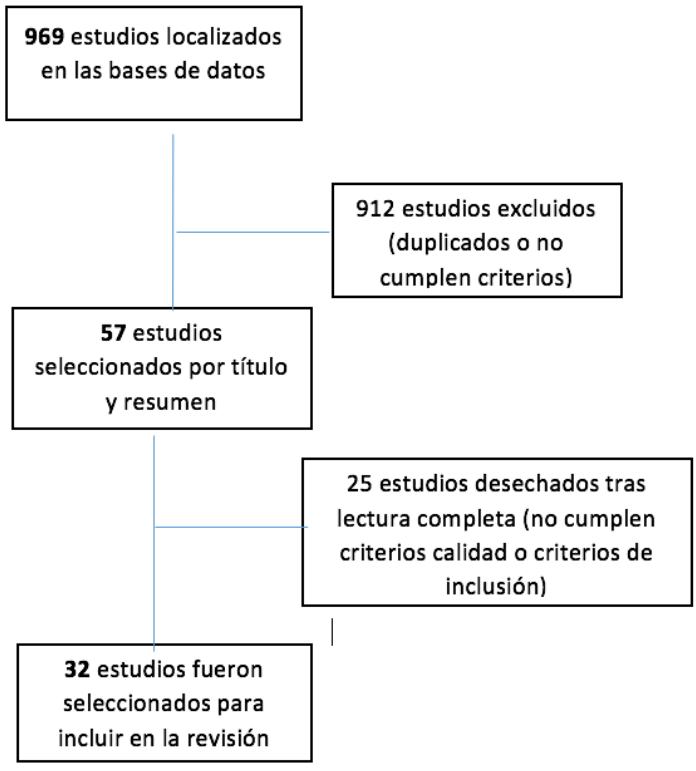

Figura 1. Procedimiento de selección, exclusión y número de estudios seleccionados.

Scopus: 489 resultados localizados; PsycINFO: 286 resultados localizados; Psicodoc: 83 resultados localizados; Medline: 111 resultados localizados. 


\section{Características de los estudios inclui- dos}

De los treinta y dos trabajos empíricos incluidos en la revisión, más de la mitad de ellos confirman que el consumo (o exposición) de algún tipo de sustancia, se trata de un factor de riesgo en el desarrollo de conductas delictivas, en concreto, un $78 \%$ de los estudios encontrados tras pasar un filtro de calidad y con un diseño de investigación que permitía la estimación del riesgo relativo.

Un $30 \%$ de los estudios se centran exclusivamente en la relación entre el alcohol y el delito, de estos artículos todos excepto uno encuentran que el consumo actual o pasado es un factor de riesgo de conductas delictivas. Un $24 \%$ se centra en el estudio de la relación con el consumo de cannabis o marihuana, y todos confirman que su consumo es un factor de riesgo. Un $6 \%$ hacía referencia a la relación entre el delito y el consumo inadecuado de fármacos y el resto estaban referidos al policonsumo (alcohol, tabaco, marihuana, cocaína) u otras sustancias (heroína, cocaína, anabolizantes). Para finalizar, en cuanto al diseño que presentan los estudios incluidos, veintiséis tienen un diseño longitudinal, el resto presentan un diseño de caso-control, transversal o prospectivo.

\section{Discusión}

El propósito que se planteó para esta revisión consistió en conocer de qué forma influye el consumo de sustancias en el desarrollo de conductas delictivas. Como se ha podido observar en el Apéndice A, los resultados de los estudios indican a grandes rasgos que el trastorno o dependencia severa (Barrett et al., 2014; McKinlay et al., 2014; Pullmann, 2010) y el consumo de varias sustancias incrementa el riesgo de participación delictiva (Marel et al., 2013) y se relaciona además con la severidad de las agresiones, específicamente de las agresiones sexuales en aquellos que las cometen (Yeater et al., 2012). A excepción de dos trabajos que no encontraron asociación significativa del consumo de sustancias ilícitas como predictor o factor de riesgo de conductas delictivas en general (Carney et al., 2013; Hunter et al., 2014).
Si nos centramos en los trabajos que estudian el consumo de cannabis, parece representar un riesgo para conductas violentas tanto en muestras de población control, cuando se consume de forma crónica (Brook et al., 2014), como en muestras de población clínica, siendo esta sustancia en esta población, la droga más relacionada con la conducta violenta (Carabellese et al., 2013). En concreto, se ha relacionado con crímenes contra la propiedad y crímenes relacionados con el tráfico de drogas (Green et al., 2010; Pedersen y Skarhamar, 2010). El estudio de Green et al. (2010) afirma que el consumo de esta sustancia aumenta 1.5 veces el riesgo de cometer crímenes contra la propiedad y 2.4 veces el riesgo de delitos relacionados con drogas, indicando que, sin embargo, no es un factor de riesgo para delitos violentos. De acuerdo con estos resultados, White et al. (2013) confirman que el consumo de marihuana se asocia al decrecimiento de la conducta agresiva.

En cuanto a los trabajos que se centran exclusivamente en el estudio de la relación entre el delito y el alcohol, indican que presentar problemas de consumo de la sustancia representa: un factor de riesgo de conducta agresiva (White et al., 2013), un aumento del riesgo de delincuencia violenta de hasta cinco veces (Dietze et al., 2013; Maldonado-Molina et al., 2011), riesgo de ser arrestado por la comisión de algún tipo de delito (Boden et al., 2013; Green et al., 2011; Jennings et al., 2015; Reingle, Jennings, Lynne-Landsman et al., 2013; Salom et al., 2014; Terranova et al., 2013) o poseer armas y cometer delitos con ellas (Brook et al., 2014). Concretamente, Boden et al. (2013) indican que, en delitos relacionados con conductas impulsivas, el alcohol tiene un gran peso. En cuanto al riesgo de delitos sexuales, no se considera el consumo de alcohol como factor predictivo (Davis et al., 2015).

Otros estudios concluyen que no es una conducta de riesgo de encarcelamiento el consumo de alcohol y marihuana (Reingle, Jennings y Komro, 2013), sin embargo, sería conveniente tener en cuenta que no todos aquellos que cometen delitos terminan entre rejas.

En relación a la exposición prenatal a sustancias como posibles factores de riesgo o predictores de conductas delictivas, un estudio indica que la exposición a la cocaí- 
na durante el embarazo es un factor de riesgo para el desarrollo de conductas delictivas (Lambert et al., 2013). Richardson et al. (2015) confirman que es la exposición durante el primer trimestre la que se asocia como factor de riesgo. Por otro lado, Gerteis et al. (2011), indican que no es un factor de riesgo la exposición a la cocaína, sino, la exposición al tabaco. No obstante, D'Onofrio et al. (2012) con una muestra significativamente mayor, concluyen que la exposición al tabaco no es un factor de riesgo de conducta delictiva cuando se controlan variables familiares y ambientales.

En cuanto a la toma de medicamentos recetados de forma inadecuada, los resultados indican que se puede considerar como un factor de riesgo para involucrarse en peleas (hasta dos veces más riesgo), aunque para la comisión de delitos no es uno de los factores que más predice este tipo de conductas (Drazdowski et al., 2015; Tucker et al., 2015). Otras sustancias como la Vareniclina y los AAS, no se asociaron como factores de riesgo (Lundholm et al., 2015; Molero et al., 2015).

Con respecto al tipo de asociación entre el consumo y el delito, sólo uno de los trabajos incluidos ha encontrado una relación recíproca entre el consumo y la delincuencia entre los catorce y quince años (McAdams et al., 2014), pero otro estudio Maldonado-Molina et al. (2011) indica que la violencia no es un factor de riesgo significativo para el consumo de alcohol.

Es de destacar que la gran mayoría de los estudios se centran en el alcohol y marihuana, en el futuro sería interesante el estudio de la influencia del consumo de algunas de las "nuevas" drogas o drogas sintéticas y su influencia en conductas violentas o delictivas, debido al aumento de su consumo actual, y su posible implicación como factor de riesgo. También podría resultar útil el análisis de los últimos estudios que traten de confirmar como factores de riesgo otras variables, como serían las variables familiares, sociales e incluso ambientales.

Otro punto importante que se ha de mencionar son las limitaciones que puede presentar esta revisión. En primer lugar, señalar el posible sesgo de información, debido a que algunos artículos no incluidos en las bases consultadas pueden haber quedado fuera de la revisión. Por otra parte, el uso de autoinformes a la hora de extraer información de la muestra de algunos artículos incluidos puede provocar que estos datos no se correspondan al cien por cien con la realidad.

Con toda esta síntesis de los resultados de los estudios, concluimos que en los últimos cinco años las investigaciones han confirmado que el consumo de sustancias (alcohol y cannabis) y el policonsumo (sobre todo en la etapa adolescente), representan un riesgo en el desarrollo de conductas delictivas o para la severidad del delito, pero sin embargo no todas las sustancias se asocian con todos los tipos de delito. Por ejemplo, el alcohol es un factor de riesgo para delitos impulsivos y violentos, mientras que el consumo de cannabis representa un riesgo de conductas violentas únicamente en población clínica, y con delitos contra la propiedad y de drogas en población general. Además, la exposición intrauterina a sustancias como la cocaína también es un factor de riesgo para el desarrollo de conductas criminales más tarde, además de las consecuencias para la salud del bebé. Y, por último, los estudios que aquí constan, no encuentran consenso en que exista una relación recíproca entre el consumo y el delito.

En definitiva, es importante señalar que conocer si la variable de consumo de sustancias representa un factor de riesgo real para el desarrollo de conductas delictivas, que era el objetivo de la revisión, resulta útil para el desarrollo de programas preventivos del crimen, y programas de actuación en centros penitenciarios. Además, no sólo podría tener el propósito de prevención del delito, sino que sería útil para la promoción de la salud y calidad de vida de la población adolescente y joven. 


\section{Referencias}

Barrett, E. L., Teesson, M. y Mills, K. L. (2014). Associations between substance use, post-traumatic stress disorder and the perpetration of violence: A longitudinal investigation. Addictive Behaviors, 39, 1075-1080.

https://doi.org/10.1016/j.addbeh.2014.03.003

Brook, J. S., Lee, J. Y., Finch, S. J. y Brook, D. W. (2014). Developmental trajectories of marijuana use from adolescence to adulthood: Relationship with using weapons including guns. Aggressive Behavior, 40, 229-237. https://doi.org/10.1002/ab.21520

Carabellese, F., Candelli, C., Martinelli, D., La Tegola, D. y Catanesi, R. (2013). Cannabis use and violent behaviour: A psychiatric patients' cohort study in Southern Italy. Rivista di Psichiatria, 48, 43-50. https://doi.org/10.1708/1228.13614

Carney, T., Myers, B. J., Louw, J., Lombard, C. y Flisher, A. J. (2013). The relationship between substance use and delinquency among high-school students in Cape Town, South Africa. Journal of Adolescence, 36, 447-455. https://doi.org/10.1016/j.adolescence.2013.01.004

Boden, J. M., Fergusson, D. M. y Horwood, L. J. (2013). Alcohol misuse and criminal offending: Findings from a 30-year longitudinal study. Drug and Alcohol Dependence, 128, 30-36. https://doi.org/10.1016/j.drugalcdep.2012.07.014

Davis, K. C., Danube, C. L., Stappenbeck, C. A., Norris, J. y George, W. H. (2015). Background predictors and event-specific characteristics of sexual aggression incidents: The roles of alcohol and other factors. Violence against Women, 21(8), 997-1017. https://doi.org/10.1177/1077801215589379

Dietze, P., Jenkinson, R., Aitken, C., Stoové, M., Jolley, D., Hickman, M. y Kerr, T. (2013). The relationship between alcohol use and injecting drug use: Impacts on health, crime and wellbeing. Drug and Alcohol Dependence, 128, 111-115. https://doi.org/10.1016/j.drugalcdep.2012.08.013

D’Onofrio, B. M., Van Hulle, C. A., Goodnight, J. A., Rathouz, P. J. y Lahey, B. B. (2012). Is maternal smoking during pregnancy a causal environmental risk factor for adolescent antisocial behavior? Testing etiological theories and assumptions. Psychological Medicine, 42, 1535-1545. https://doi.org/10.1017/S0033291711002443

Drazdowski, T. K., Jäggi, L., Borre, A. y Kliewer, W. L. (2015). Use of prescription drugs and future delinquency among adolescent offenders. Journal of Substance Abuse Treatment, 48, 28-36. https://doi.org/10.1016/j.jsat.2014.07.008

Dubow, E. F., Huesmann, L. R., Boxer, P. y Smith, C. (2014). Childhood predictors and age 48 outcomes of self-reports and official records of offending. Criminal Behaviour and Mental Health, 24, 291304. https://doi.org/10.1002/cbm.1929

Ford, J. A. (2008). Nonmedical prescription drug use and delinquency: An analysis with a national sample. Journal of Drug Issues, 38, 493-516. https://doi.org/10.1177/002204260803800206

Gatti, U., Soellner, R., Schadee, H., Verde, A. y Rocca, G. (2013). Effects of delinquency on alcohol use among juveniles in Europe: Results from the ISRD2 study. European Journal on Criminal Policy and Research, 19, 153-170. https://doi.org/10.1007/s10610-013-9202-x

Gerteis, J., Chartrand, M., Martin, B., Cabral, H. J., Rose-Jacobs, R., Crooks, D. y Frank D. A. (2011). Are there effects of intrauterine cocaine exposure on delinquency during early adolescence? A preliminary report. Journal of Developmental \& Behavioral Pediatrics, 32, 393-401. https://doi.org/10.1097/DBP.0b013e318218d9f2

Green, K. M., Doherty, E. E., Stuart, E. A. y Ensminger, M. E. (2010). Does heavy adolescent marijuana use 
lead to criminal involvement in adulthood? Evidence from a multiwave longitudinal study of urban African Americans. Drug and Alcohol Dependence, 112, 117-125. https://doi.org/10.1016/j.drugalcdep.2010.05.018

Green, K. M., Doherty, E. E., Zebrak, K. A. y Ensminger, M. E. (2011). Association between adolescent drinking and adult violence: evidence from a longitudinal study of urban African Americans. Journal of Studies on Alcohol and Drugs, 72, 701-710.

Hunter, S. B., Miles, J. N., Pedersen, E. R., Ewing, B. A. y D'Amico, E. J. (2014). Temporal associations between substance use and delinquency among youth with a first-time offense. Addictive Behaviors, 39 , 1081-1086. https://doi.org/10.1016/j.addbeh.2014.03.002

Instituto Nacional de Estadística (2015). Estadística de condenados: Menores [Conviction Statistics: Juveniles]. Recuperado de http://www.ine.es/dyngs/INEbase/es/operacion.htm? $\mathrm{c}=$ Estadistica $\mathrm{C} \& \mathrm{cid}=1254736176795$ \&menu $=$ ulti Datos\&idp $=1254735573206$

Jennings, W. G., Piquero, A. R., Roque, M. y Farrington, D. P. (2015). The effects of binge and problem drinking on problem behavior and adjustment over the life course: Findings from the cambridge study in delinquent development. Journal of Criminal Justice, $\quad 43, \quad 453-463$. https://doi.org/10.1016/j.jcrimjus.2015.09.002

Lambert, B. L., Bann, C. M., Bauer, C. R., Shankaran, S., Bada, H. S., Lester, B. M., ... Higgins, R. D. (2013). Risk-taking behavior among adolescents with prenatal drug exposure and extrauterine environmental adversitiy. Journal of Developmental \& Behavioral Pediatrics, 34, 669-679. https://doi.org/10.1097/01.DBP.0000437726.16588. e2

Lundholm, L., Frisell, T., Lichtenstein, P. y Långström, N. (2015). Anabolic androgenic steroids and violent offending: confounding by polysubstance abuse among 10365 general population men. Addiction, 110, 100-108. https://doi.org/10.1111/add.12715

Maldonado-Molina, M. M., Reingle, J. M. y Jennings, W. G. (2011). Does alcohol use predict violent behaviors? The relationship between alcohol use and violence in a nationally representative longitudinal sample. Youth Violence and Juvenile Justice, 9, 99-111. https://doi.org/10.1177/1541204010384492

Marel, C., Mills, K. L., Darke, S., Ross, J. Slade, T., Burns, L. y Teesson, M. (2013). Static and dynamic predictors of criminal involvement among people with heroin dependence: Findings from a 3-year longitudinal study. Drug and Alcohol Dependence, 133 , 600-606. https://doi.org/10.1016/j.drugalcdep.2013.08.003

McAdams, T. A., Salekin, R. T., Marti, C. N., Lester, W. S. y Barker, E. D. (2014). Co-ocurrence of antisocial behavior and substance use: Testing for sex differences in the impact of older male friends, low parental knowledge and friends' delinquency. Journal of Adolescence, 37, 247-256. https://doi.org/10.1016/j.adolescence.2014.01.001

McKinlay, A., Corrigan, J., Horwood, L. J. y Fergusson, D. M. (2014). Substance abuse and criminal activities following traumatic brain injury in childhood, adolescence, and early adulthood. The Journal of Head Trauma Rehabilitation, 29(6), 498506.

https://doi.org/10.1097/HTR.0000000000000001

Miller, P. G., Butler, E., Richardson, B., Staiger, P. K. youssef, G. J., Macdonald, J. A., ...Olsson, C. A. (2016). Relationships between problematic alcohol consumption and delinquent behaviour from adolescence to young adulthood. Drug and Alcohol Review, 35, 317-325. https://doi.org/10.1111/dar.12345

Ministerio de Sanidad, Servicios Sociales e Igualdad (2013). Encuesta Nacional de Salud. España 
2011/12. Serie Informes monográficos $n^{o} 1$. Consumo de alcohol [National Health Statistics. Spain 2011/12. Series Reports $n^{\mathbf{o}}$ 1. Alcohol Consumption]. Recuperado de http://www.msssi.gob.es/estadEstudios/estadisticas/ encuestaNacional/encuestaNac2011/informesMono graficos/ENSE2011_12_MONOGRAFICO_1_ALC OHOL4.pdf

Molero, Y., Lichtenstein, P., Zetterqvist, J., Gumpert, C. H. y Fazel, S. (2015). Varenicline and risk of psychiatric condictions, suicidal behavior, criminal offending, and transport accidents and offences: population, based cohort study. The British Medical Journal, 350 , https://doi.org/10.1136/bmj.h2388

Montgomery, K. L., Thomson, S. J. y Barczyk, A. N. (2011). Individual and relationship factor associated with delinquency among throwaway adolescents. Children and Youth Services Review, 33, 11271133. https://doi.org/10.1016/j.childyouth.2011.02.005

Nordstrom, B. R. y Dackis, C. A. (2011). Drugs and crime. Journal of Psychiatry \& Law, 39, 663-687. https://doi.org/10.1177/009318531103900407

Observatorio Español de las Drogas y las Toxicomanías (2015). Estadísticas 2015: Alcohol, tabaco y drogas ilegales en España [Statistics 2015: Alcohol, Tobacco and Illegal Drugs in Spain]. Recuperado de http://www.pnsd.msssi.gob.es/profesionales/sistema sInformacion/informesEstadisticas/pdf/ESTADISTI CAS_2015.pdf

Observatorio Europeo de las Drogas y las Toxicomanías (2016). Informe Europeo sobre drogas. Tendencias $y$ novedades [European Drug Report. Trends and Developments]. Recuperado de http://www.emcdda.europa.eu/system/files/publicati ons/2637/TDAT16001ESN.pdf

Oficina Europea de Estadística. (2010). Crime trends in detail.

Recuperado

de http://ec.europa.eu/eurostat/statistics-

explained/index.php/Crime_trends_in_detail

Organización Mundial de la Salud. (2014). Global status report on alcohol and health. Recuperado de http://apps.who.int/iris/bitstream/10665/112736/1/9 789240692763 eng.pdf

Pedersen, W. y Skardhamar, T. (2010). Cannabis and crime: Findings from a longitudinal study. Addiction, 105, 109-118. https://doi.org/10.1111/j.1360-0443.2009.02719.x

Pita, S., Vila, M. T. y Carpente, J. (1997). Determinación de factores de riesgo [Determination of Risk Factors]. Cadernos de Atención Primaria, 4(2), 7578.

Pope, H. G., Kouri, E. M. y Hudson, J. I. (2000). Effects of supraphysiologic doses of testosterone on mood and aggression in normal men: A randomized controlled trial. Archives of General Psychiatry, 57, 133-140. https://doi.org/10.1001/archpsyc.57.2.133

Pullmann, M. D. (2010). Predictors of criminal charges for youth in public mental health during the transition to adulthood. Journal of Child and Family Studies, 19, 483-491. https://doi.org/10.1007/s10826-009-9320-8

Reingle, J. M., Jennings, W. G. y Komro, K. A. (2013). A Case-Control Study of Risk and Protective Factors for Incarceration Among Urban Youth. Journal of Adolescent Health, 53, 471-477. https://doi.org/10.1016/j.jadohealth.2013.05.008

Reingle, J. M., Jennings, W. G., Lynne-Landsman, S. D., Cottler, L. B. y Maldonado-Molina, M. M. (2013). Toward an understanding of risk and protective factors for violence among adolescent boys and men: A longitudinal analysis. Journal of Adolescent Health, 52, 493-498. https://doi.org/10.1016/j.jadohealth.2012.08.006
Reingle, J. M., Jennings, W. G. y Maldonado-Molina, M. M. (2012). Risk and protective factors for 
trajectories of violent delinquency among a nationally representative sample of early adolescents. Youth Violence and Juvenile Justice, 10 , https://doi.org/10.1177/1541204011431589

Richardson, G. A., Goldschmidt, L., Larkby, C. y Day, N. L. (2015). Effects of prenatal cocaine exposure on adolescent development. Neurotoxicology and Teratology, 49, 41-48. https://doi.org/10.1016/j.ntt.2015.03.002

Salom, C. L., Betts, K. S., Williams, G. M., Najman, J. M., Scott, J. G. y Alati, R. (2014). Do young people with comorbid mental and alcohol disorders experience worse behavioural problems? Psychiatry Research, 219, 372-379. https://doi.org/10.1016/j.psychres.2014.05.056

Secretaría General de Instituciones Penitenciarias (2016). Estadística penitenciaria. Población reclusa por sexo y edad (noviembre) [Penitentiary Statistics. Population Recluse by Sex and Age (November)]. Recuperado de http://www.institucionpenitenciaria.es/web/portal/d ocumentos/

Swahn, M. H. y Donovan, J. E. (2004). Correlates and predictors of violent behavior among adolescent drinkers. Journal of Adolescence Health, 34, 480492.

https://doi.org/10.1016/j.jadohealth.2003.08.018

Terranova, C., Tucci, M., Sartore, D., Cavarzeran, F., Di Pietra, L., Barzon, L. ... Ferrara, S. D. (2013). GABA receptors, alcohol dependence and criminal behavior. Journal of Forensic Sciences, 58, 12281232. https://doi.org/10.1111/1556-4029.12201

Tucker, J. S., Ewing, B. A., Miles, J. N. V., Shih, R. A., Pedersen, E. R. y D'Amico, E. J. (2015). Predictors and consequences of prescription drug misuse during middle school. Drug and Alcohol Dependence, $\quad 156, \quad 254-260$. https://doi.org/10.1016/j.drugalcdep.2015.09.018

Von Elm, E., Altman, D. G., Egger, M., Pocock, S. J., GØtzsche, P. C. y Vandenbroucke, J. P. (2008). Declaración de la iniciativa STROBE (Strengthening the reporting of observational studies in epidemiology): Directrices para la comunicación de estudios observacionales [The Strengthening the Reporting of Observational Studies in Epidemiology Statement (STROBE): Guidelines for Reporting Observational Studies]. Gaceta Sanitaria, 22, 144150.

White, H. R., Fite, P., Pardini, D., Mun, E. Y. y Loeber, R. (2013). Moderators of the dynamic link between alcohol use and aggressive behavior among adolescent males. Journal of Abnormal Child Psychology, 41, 211-222. https://doi.org/10.1007/s10802-012-9673-0

Yeater, E. A., Lenberg, K. L. y Bryan, A. D. (2012). Predictors of sexual aggression among male juvenile offenders. Journal of Interpersonal Violence, $27, \quad 1242-1258$. https://doi.org/10.1177/0886260511425243 


\section{Apéndice}

Características de los estudios seleccionados y sus principales resultados en relación al consumo como factor de riesgo de la delincuencia

\begin{tabular}{|c|c|c|c|c|c|c|}
\hline $\begin{array}{c}\text { Autor/es } \\
\text { (año) }\end{array}$ & Objetivo & $\mathbf{N}$ & $\begin{array}{c}\text { Características } \\
\text { de la muestra }\end{array}$ & Variables & Diseño & Resultados \\
\hline $\begin{array}{l}\text { Barrett, } \\
\text { Teesson y } \\
\text { Mills (2014) }\end{array}$ & $\begin{array}{l}\text { Examinar los } \\
\text { cambios en el } \\
\text { abuso de } \\
\text { sustancias y } \\
\text { sintomatología } \\
\text { postraumática con } \\
\text { la comisión de } \\
\text { delitos violentos }\end{array}$ & 102 & $\begin{array}{l}\text { Mujeres y } \\
\text { hombres } \\
\text { Consumidores } \\
\text { y con síntomas } \\
\text { postraumáticos } \\
\text { Australia }\end{array}$ & $\begin{array}{l}\text { Delincuencia, } \\
\text { severidad de los } \\
\text { síntomas } \\
\text { postraumáticos, } \\
\text { depresión ansiedad, } \\
\text { consumo y } \\
\text { dependencia de } \\
\text { sustancias }\end{array}$ & $\begin{array}{l}\text { Longitudinal } \\
\text { ( } 9 \text { meses de } \\
\text { seguimiento) }\end{array}$ & $\begin{array}{l}\text { Aquellos que } \\
\text { tienen una } \\
\text { dependencia a } \\
\text { las sustancias } \\
\text { más severa son } \\
\text { más propensos a } \\
\text { cometer delitos } \\
\text { violentos }\end{array}$ \\
\hline $\begin{array}{l}\text { Boden, } \\
\text { Fergusson y } \\
\text { Hordwood } \\
(2013)\end{array}$ & $\begin{array}{l}\text { Examinar } \\
\text { asociaciones } \\
\text { entre abuso y } \\
\text { dependencia del } \\
\text { alcohol y la } \\
\text { conducta criminal }\end{array}$ & 1265 & $\begin{array}{l}\text { Mujeres y } \\
\text { hombres } \\
\text { Nueva Zelanda }\end{array}$ & $\begin{array}{l}\text { Delitos cometidos, } \\
\text { abuso/dependencia de } \\
\text { alcohol }\end{array}$ & $\begin{array}{l}\text { Longitudinal } \\
\text { (30 años de } \\
\text { seguimiento) }\end{array}$ & $\begin{array}{l}\text { Síntomas de } \\
\text { dependencia } \\
\text { factor de riesgo } \\
\text { de algunos tipos } \\
\text { de delito }\end{array}$ \\
\hline $\begin{array}{l}\text { Brook, Lee, } \\
\text { Finch y Brook } \\
(2014)\end{array}$ & $\begin{array}{l}\text { Estudiar la } \\
\text { asociación entre } \\
\text { las trayectorias de } \\
\text { uso de marihuana } \\
\text { y otros predictores } \\
\text { de violencia con el } \\
\text { uso de armas }\end{array}$ & 838 & $\begin{array}{l}\text { Mujeres y } \\
\text { hombres } \\
\text { Afroamericanos } \\
\text { y Puerto Rico }\end{array}$ & $\begin{array}{l}\text { Demográficas, } \\
\text { delincuencia, consumo } \\
\text { de alcohol, nivel de } \\
\text { estudios, consumo de } \\
\text { marihuana, uso y } \\
\text { posesión de armas }\end{array}$ & $\begin{array}{l}\text { Prospectivo } \\
\text { Longitudinal }\end{array}$ & $\begin{array}{l}\text { El consumo de } \\
\text { marihuana puede } \\
\text { dar lugar a } \\
\text { comportamientos } \\
\text { violentos antes } \\
\text { de la etapa adulta }\end{array}$ \\
\hline $\begin{array}{l}\text { Carabellese, } \\
\text { Candelli, } \\
\text { Martinelli, La } \\
\text { Tegola y } \\
\text { Catanesi } \\
(2013)\end{array}$ & $\begin{array}{l}\text { Identificar y } \\
\text { analizar la } \\
\text { relación entre el } \\
\text { consumo de } \\
\text { cannabis y la } \\
\text { conducta violenta }\end{array}$ & 1582 & $\begin{array}{l}\text { Mujeres y } \\
\text { hombres } \\
\text { Muestra clínica } \\
\text { Edad media: } 45 \\
\text { Italia }\end{array}$ & $\begin{array}{l}\text { Conducta violenta, } \\
\text { datos familiares, } \\
\text { consumo de cannabis }\end{array}$ & Retrospectivo & $\begin{array}{l}\text { El consumo de } \\
\text { cannabis } \\
\text { representa un } \\
\text { riesgo para la } \\
\text { conducta violenta }\end{array}$ \\
\hline $\begin{array}{l}\text { Carney, } \\
\text { Myers, Louw, } \\
\text { Lombard y } \\
\text { Flisher } \\
(2013)\end{array}$ & $\begin{array}{l}\text { Examinar los } \\
\text { predictores } \\
\text { significativos de } \\
\text { conductas } \\
\text { delictivas } \\
\text { centrándose en la } \\
\text { relación con el } \\
\text { alcohol y abuso } \\
\text { de drogas }\end{array}$ & 638 & $\begin{array}{l}\text { Mujeres y } \\
\text { hombres } \\
\text { Sudáfrica }\end{array}$ & $\begin{array}{l}\text { Tipo de conducta } \\
\text { delictiva, consumo de } \\
\text { drogas y alcohol, } \\
\text { consumo de tabaco, } \\
\text { demográficas, } \\
\text { escolares }\end{array}$ & $\begin{array}{l}\text { Prospectivo } \\
\text { Longitudinal } \\
\text { (4 años de } \\
\text { seguimiento) }\end{array}$ & $\begin{array}{l}\text { Consumo de } \\
\text { alcohol, tabaco y } \\
\text { drogas no son } \\
\text { predictores } \\
\text { significativos de } \\
\text { conducta delictiva } \\
\text { Fumar tabaco } \\
\text { modifica el riesgo } \\
\text { de delincuencia } \\
\text { futura. }\end{array}$ \\
\hline
\end{tabular}




$\begin{array}{ll}\text { Davis, } & \text { Examinar las } \\ \text { Danube, } & \text { características de } \\ \text { Stappenbeck, } & \text { los episodios de } \\ \text { Norris y } & \text { abuso sexual y } \\ \text { George } & \text { sus predictores. } \\ (2015) & \end{array}$

\begin{tabular}{|c|c|}
\hline $\begin{array}{l}\text { Dietze et al. } \\
(2013)\end{array}$ & $\begin{array}{l}\text { Explorar los } \\
\text { efectos del } \\
\text { consumo alcohol } \\
\text { sobre la salud, el } \\
\text { crimen y la } \\
\text { satisfacción. }\end{array}$ \\
\hline $\begin{array}{l}\text { D'Onofrio, } \\
\text { Van Hulle, } \\
\text { Goodnight, } \\
\text { Rathouz y } \\
\text { Lahey (2012) }\end{array}$ & $\begin{array}{l}\text { Examinar la } \\
\text { asociación entre } \\
\text { fumar durante el } \\
\text { embarazo y } \\
\text { conducta ofensiva } \\
\text { en la } \\
\text { adolescencia. }\end{array}$ \\
\hline $\begin{array}{l}\text { Drazdowski, } \\
\text { Jäggi, Borre } \\
\text { y Kliewer } \\
(2015)\end{array}$ & $\begin{array}{l}\text { Conocer el perfil } \\
\text { de los } \\
\text { adolescentes } \\
\text { NMUPD y cuál es } \\
\text { su contribución en } \\
\text { la predicción de } \\
\text { delincuencia } \\
\text { futura. }\end{array}$ \\
\hline
\end{tabular}

Conocer si el nivel

de exposición

intrauterina a la

Gerteis et al.

(2011)

cocaína está

asociado con

conducta delictiva

adolescente.

Probar la

Green,

Doherty,

Stuart y

Ensminger

(2010)

Green,

Doherty,

Zebrak y

Esminger

(2011) asociación entre

el consumo

adolescente de

marihuana y el

crimen.

Examinar la relación entre la frecuencia de consumo de alcohol de los
688

hombres

Edad: 18-40

Consumidores

de sustancias

Australia

\section{E.E.U.U.}

Australa

Mujeres y

6066 hombres

E.E.U.U.

Hombres

Adolescentes

Culpables en

delitos serios

E.E.U.U.

93

Historial de abuso

sexual, consumo de alcohol, agresividad e impulsividad,

actitudes, variables

sociales y de relación.

Salud, crimen,

satisfacción, consumo

de alcohol, factores de confusión.

Conducta antisocial, conducta criminal, frecuencia consumo de tabaco de la madre, variables familiares.

Delincuencia futura, historia criminal, uso de drogas, salud mental.

\section{Mujeres y}

hombres

La mitad había

estado

expuesto a la

cocaína

E.E.U.U.

\section{Mujeres y}

Hombres

Raza negra

E.E.U.U.

Mujeres y

hombres

702

Afroamericanos

E.E.U.U.

Conducta delictiva, exposición intrauterina a la cocaína y otras sustancias.

Delincuencia, consumo de marihuana.

Frecuencia del consumo de alcohol, violencia en adultos, crimen no violento, variables mediadoras,
Prospectivo

Longitudinal

(3 meses de

seguimiento)

(1)

Longitudinal

Longitudinal

tabaco de la

madre no se

relaciona con la

aparición de

conductas

antisociales.

NMUPD predictor significativo de la delincuencia

Longitudinal

futura pero no

más allá de otros

predictores más

potentes.

Prospectivo Longitudinal (12 años de seguimiento)

Longitudinal (36 años de seguimiento) variables de
La exposición intrauterina a la cocaína no se asocia como factor de riesgo.

Consumo intensivo aumenta el riesgo de cometer crímenes contra la propiedad y relacionados con las drogas.
El consumo frecuente de alcohol entre los adolescentes predice un aumento del 
adolescentes y el desarrollo de

violencia en adultos.

$\begin{array}{ll}\text { Hunter, } & \text { Examinar la } \\ \text { Miles, } & \text { asociación } \\ \text { Pedersen, } & \text { temporal entre la } \\ \text { Ewing y } & \text { celincuencia y el } \\ \text { D’Amico } & \text { consumo de } \\ \text { (2014) } & \text { sustancias. }\end{array}$

Prevalencia de los problemas de

$\begin{array}{ll}\text { Jennings, } & \text { bebida y su } \\ \text { Piquero, } & \text { relación }\end{array}$

Rocque y

Farrington

(2015)

relación con la

delincuencia

violenta y no-

violenta.

\section{Examinar la}

influencia de la

exposición

Lambert et al. prenatal a

(2013)

sustancias y el

riesgo de

conducta delictiva

adolescente.

Lundholm,

Frisell,

Examinar la

Lichtenstein y el uso AAS y el

Langström crimen violento

(2015) controlando otros

factores de riesgo.

Maldonado-

Molina,

Reingle y

Jennings

(2011)

\section{Examinar la \\ relación entre el \\ alcohol y la \\ violencia}

$\begin{array}{ll} & \\ & \text { Examina la } \\ & \text { relación entre } \\ \text { predictores } \\ \text { Marel et al. } & \text { estáticos y } \\ (2013) & \text { dinámicos del } \\ & \text { comportamiento } \\ & \text { criminal. }\end{array}$

correspondencia

$\begin{array}{ll}\text { Mujeres y } & \text { Delincuencia, } \\ \text { hombres } & \text { consumo de } \\ \text { Cometieron su } & \text { sustancias, } \\ \text { primer delito } & \text { consecuencias } \\ \text { entre los 14 y } & \text { negativas del } \\ \text { los 18 años. } & \text { consumo. }\end{array}$

Delincuencia/ofensas, consumo de alcohol, factores individuales, problemas de ajuste.

Arrestos y abuso de sustancias, conducta sexual, control inhibitorio exposición prenatal a las drogas, factores de riesgo sociodemográfico y ambiental.

Hombres

Gemelos

10365 Suecia

Consumo de AAS

Edad: 20-47.

Mujeres y

hombres

E.E.U.U.

Edad media: 15

Crimen violento, consumo AAS,

consumo de otras sustancias.

Conducta violenta a lo largo del tiempo, consumo problemático de alcohol a lo largo

del tiempo,

covariables

615

Mujeres y

hombres

Dependientes

de heroína

Australia. mental, demográficas, participación delictiva. factores ambientales,

riesgo a ser

arrestado por

delitos violentos.

No encuentran

evidencia de

asociación

Transversal temporal significativa entre el consumo y el delito.

Prospectivo Longitudinal (30 años de seguimiento)

Los problemas con la bebida son un predictor significativo de la delincuencia.

La exposición prenatal a la

Prospectivo cocaína supuso un riesgo de arresto adolescente.

El consumo de AAS no supone

Transversal por sí mismo un riesgo de crimen violento.

El consumo constante de Longitudinal alcohol es un (8 años de seguimiento) factor predictivo de la violencia grave.

La intensidad y severidad del consumo de heroína y el consumo de otras drogas junto con otras variables sustancias, salud Longitudinal
(3 años de seguimiento) tiene relación

positiva con el riesgo de participación delictiva. 
Evaluar la relación

McAdams,

Salekin,

Marti, Lester

y Barker

(2014)

$3699 \quad \begin{aligned} & \text { Mujeres y } \\ & \text { hombres } \\ & \text { Reino Unido. }\end{aligned}$

Delincuencia, abuso de sustancias, amigos delincuentes, variables ambientales. adolescencia temprana.

Evaluar la

McKinlay, Corrigan, Horwood y Fergusson (2014) asociación entre daño cerebral con abuso de drogas $y$ alcohol y actividades criminales.

\section{Examinar la}

Molero,

Lichtenstein,

Zetterqvist,

Gumpert y Fazel (2015) asociación entre la vareniclina y la incidencia de condiciones psiquiátricas, suicidio y crimen.

Examinar asociación entre

Pedersen y Skarhamar (2010) el consumo de cannabis durante la juventud y cargos criminales posteriores.

Examinar la Pullmann relación entre (2010) servicios de salud mental durante la adolescencia y cargos criminales.

Mujeres y hombres Ingresados en instituciones

Edad: $<19$

Examinar factores de riesgo y

Reingle, Jennings y Komro (2013)

Mujeres y

hombres

2165

riesgo protección

tempranos y el

riesgo de encarcelamiento.
Mujeres y

hombres

Nueva Zelanda.

Mujeres y hombres

69757

Edad: $>15$

Tratados con

vareniclina

Suecia.

Mujeres y

hombres

Noruega.

Jóvenes en

E.E.U.U.
Entre los 13 y los

14 años la

delincuencia es

un predictor del consumo,

Transversal

mientras que

entre los 14 y los

15 tienen una

relación

recíproca.

Los hallazgos

apoyan que el

Dependencia alcohol y drogas,

conducta criminal y

daño cerebral.

Longitudinal (25 años de seguimiento) de sustancias incrementa el riesgo de conducta criminal.

Tratamiento con vareniclina, conducta suicida, variables psiquiátricas, crimen, consumo de sustancias.

Longitudinal (3 años de seguimiento)

Sin evidencia de asociación causal entre la toma de vareniclina con las conductas estudiadas.

\section{El consumo de} cannabis está asociado con un incremento de

Crimen, consumo de sustancias, conductas problema, variables familiares, socioeconómicas y escolares.

Longitudinal riesgo de crimen (14 años de relacionado con seguimiento) las drogas, pero no con otros tipos de crimen.

El diagnóstico de adicción a sustancias está

Delincuencia, psicopatología, género, servicios recibidos.

Longitudinal (9 años de seguimiento) relacionado con un aumento del riesgo de conducta delictiva.

Encarcelamiento, agresión, consumo de sustancias, contexto, demográficas, conducta joven y escolar.
El consumo de marihuana y alcohol no son

Caso-Control factores de riesgo tempranos importantes para la encarcelación. 


\begin{tabular}{|c|c|c|c|c|c|c|}
\hline $\begin{array}{l}\text { Reingle, } \\
\text { Jennings, } \\
\text { Lynne- } \\
\text { Landsman, } \\
\text { Cottler y } \\
\text { Maldonado- } \\
\text { Molina (2013) }\end{array}$ & $\begin{array}{l}\text { Identificar factores } \\
\text { de riesgo y de } \\
\text { protección } \\
\text { además de } \\
\text { diferencias de } \\
\text { género en la } \\
\text { violencia seria. }\end{array}$ & 4322 & $\begin{array}{l}\text { Mujeres y } \\
\text { Hombres } \\
\text { Estudiantes } \\
\text { E.E.U.U. }\end{array}$ & $\begin{array}{l}\text { Violencia/delincuencia, } \\
\text { comunidad, familia e } \\
\text { iguales, consumo } \\
\text { drogas. }\end{array}$ & $\begin{array}{l}\text { Longitudinal } \\
\text { (14 años de } \\
\text { seguimiento) }\end{array}$ & $\begin{array}{l}\text { Consumo de } \\
\text { alcohol factor de } \\
\text { riesgo conducta } \\
\text { delictiva en } \\
\text { desistores. }\end{array}$ \\
\hline $\begin{array}{l}\text { Reingle, } \\
\text { Jennings y } \\
\text { Maldonado- } \\
\text { Molina (2012) }\end{array}$ & $\begin{array}{l}\text { Estimar } \\
\text { trayectorias de } \\
\text { violencia } \\
\text { considerando los } \\
\text { efectos de } \\
\text { múltiples } \\
\text { influencias. }\end{array}$ & 9421 & $\begin{array}{l}\text { Mujeres y } \\
\text { hombres } \\
\text { Estudiantes } \\
\text { E.E.U.U. }\end{array}$ & $\begin{array}{l}\text { Delincuencia violenta, } \\
\text { factores de riesgo } \\
\text { comunitario y de } \\
\text { pares, factores } \\
\text { individuales (drogas y } \\
\text { alcohol). }\end{array}$ & $\begin{array}{l}\text { Longitudinal } \\
\text { (14 años de } \\
\text { seguimiento) }\end{array}$ & $\begin{array}{l}\text { Consumo de } \\
\text { alcohol } \\
\text { marihuana y } \\
\text { otras drogas } \\
\text { como factor de } \\
\text { riesgo de } \\
\text { pertenecer a } \\
\text { criminales } \\
\text { desistores. }\end{array}$ \\
\hline $\begin{array}{l}\text { Richardson, } \\
\text { Goldschmidt, } \\
\text { Larkby y Day } \\
(2015)\end{array}$ & $\begin{array}{l}\text { Efectos de la } \\
\text { exposición } \\
\text { prenatal a la } \\
\text { cocaína sobre la } \\
\text { conducta } \\
\text { adolescente. }\end{array}$ & 219 & $\begin{array}{l}\text { Mujeres y } \\
\text { hombres } \\
\text { E.E.U.U. }\end{array}$ & $\begin{array}{l}\text { Conducta delictiva, } \\
\text { variables cognitivas, } \\
\text { crecimiento, consumo } \\
\text { de cocaína de la } \\
\text { madre y otras drogas. }\end{array}$ & $\begin{array}{l}\text { Longitudinal } \\
\text { (15 años de } \\
\text { seguimiento) }\end{array}$ & $\begin{array}{l}\text { Adolescentes } \\
\text { expuestos a la } \\
\text { cocaína tienen } \\
\text { más riesgo de } \\
\text { conducta } \\
\text { delictiva. }\end{array}$ \\
\hline $\begin{array}{l}\text { Salom et al. } \\
(2014)\end{array}$ & $\begin{array}{l}\text { Comprobar la } \\
\text { asociación entre } \\
\text { los problemas de } \\
\text { conducta en } \\
\text { personas con } \\
\text { problemas de } \\
\text { salud mental y el } \\
\text { alcohol. }\end{array}$ & 2314 & $\begin{array}{l}\text { Mujeres y } \\
\text { hombres } \\
\text { Australia. }\end{array}$ & $\begin{array}{l}\text { Problemas } \\
\text { conductuales, } \\
\text { comorbilidad, } \\
\text { covariables. }\end{array}$ & $\begin{array}{l}\text { Longitudinal } \\
\text { (21 años de } \\
\text { seguimiento) }\end{array}$ & $\begin{array}{l}\text { Las personas con } \\
\text { alguna } \\
\text { psicopatología } \\
\text { que consumen } \\
\text { alcohol tienen } \\
\text { dos veces más } \\
\text { riesgo de } \\
\text { cometer delitos. }\end{array}$ \\
\hline $\begin{array}{l}\text { Terranova et } \\
\text { al. (2013) }\end{array}$ & $\begin{array}{l}\text { Analizar la } \\
\text { conexión entre la } \\
\text { dependencia al } \\
\text { alcohol y la } \\
\text { conducta criminal } \\
\text { con un enfoque } \\
\text { genético- } \\
\text { ambiental. }\end{array}$ & 186 & $\begin{array}{l}\text { Hombres } \\
47 \text { convictos } \\
139 \text { no } \\
\text { convictos } \\
\text { Italia } \\
\text { Edad: } 25-70\end{array}$ & $\begin{array}{l}\text { Conducta Criminal, } \\
\text { poliformismos } \\
\text { genéticos en } \\
\text { receptores GABA, } \\
\text { consumo de alcohol, } \\
\text { variables ambientales. }\end{array}$ & Caso-control & $\begin{array}{l}\text { Problemas con el } \\
\text { alcohol junto con } \\
\text { factores } \\
\text { genéticos } \\
\text { incrementan } \\
\text { cuatro veces el } \\
\text { riesgo de } \\
\text { conducta } \\
\text { antisocial. }\end{array}$ \\
\hline $\begin{array}{l}\text { Tucker et al. } \\
(2015)\end{array}$ & $\begin{array}{l}\text { Conocer la } \\
\text { asociación entre } \\
\text { el mal uso de } \\
\text { medicamentos } \\
\text { durante la escuela } \\
\text { sobre la } \\
\text { delincuencia } \\
\text { posterior. }\end{array}$ & 12904 & $\begin{array}{l}\text { Mujeres y } \\
\text { hombres } \\
\text { Estudiantes } \\
\text { E.E.U.U. }\end{array}$ & $\begin{array}{l}\text { Funcionamiento } \\
\text { escolar (salud mental, } \\
\text { delincuencia, etc.), } \\
\text { variables familiares, } \\
\text { variables individuales. }\end{array}$ & $\begin{array}{l}\text { Longitudinal } \\
\text { (3 años de } \\
\text { seguimiento). }\end{array}$ & $\begin{array}{l}\text { Estudiantes que } \\
\text { tomaban de } \\
\text { forma } \\
\text { inadecuada los } \\
\text { medicamentos } \\
\text { tenían más de } \\
\text { riesgo de } \\
\text { involucrarse en } \\
\text { peleas. }\end{array}$ \\
\hline $\begin{array}{l}\text { White, Fite, } \\
\text { Pardini, Mun } \\
\text { y Loeber } \\
(2013)\end{array}$ & $\begin{array}{l}\text { Examinar la } \\
\text { asociación entre } \\
\text { el consumo de } \\
\text { alcohol y la } \\
\text { conducta agresiva } \\
\text { adolescente. }\end{array}$ & 971 & $\begin{array}{l}\text { Hombres } \\
\text { E.E.U.U. }\end{array}$ & $\begin{array}{l}\text { Conducta agresiva, } \\
\text { consumo de alcohol, } \\
\text { variables } \\
\text { moderadoras. }\end{array}$ & $\begin{array}{l}\text { Prospectivo } \\
\text { Longitudinal } \\
\text { (14 años de } \\
\text { seguimiento) }\end{array}$ & $\begin{array}{l}\text { Un incremento } \\
\text { del consumo de } \\
\text { alcohol está } \\
\text { asociado a un } \\
\text { incremento de la } \\
\text { conducta } \\
\text { agresiva. }\end{array}$ \\
\hline
\end{tabular}




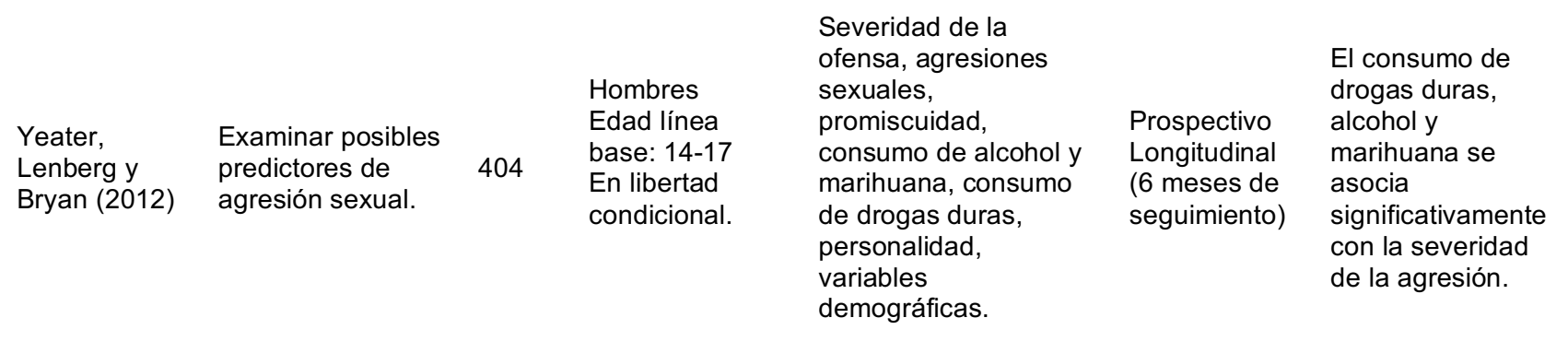

\title{
SUBSTANCE ABUSE AS A RISK FACTOR FOR CRIMINAL BEHAVIOR: A SYSTEMATIC REVIEW
}

\author{
ESTHER PÉREZ ${ }^{1}$ Y SERGIO RUIZ ${ }^{1}$
}

\section{EXTENDED SUMMARY}

\section{Introduction}

Several studies have linked crime with substance abuse variables in the past. For example, Montgomery, Thomson and Barczyk (2011) pointed out that individual factors like drugs or mental illnesses could explain about $40 \%$ of crime variance (cases of crime). The direction of this suggested association between these variables is unclear and drug use could act either as a risk factor (e.g., Swahn \& Donovan, 2004), a mediating factor (e.g., Miller et al., 2016) or a consequence of criminal activity. From this, it arises the need to review studies whose statistical analysis design focuses on checking whether substance use actually poses a risk in the occurrence of criminal behavior.

Worldwide data indicates that alcohol consumption is more than six liters per person (Organización Mundial de la Salud [OMS], 2014), and in Spain, the average age of onset of consumption dropped significantly (Ministerio de Sanidad, Servicios Sociales e Igualdad, 2013). On the other hand, data on substance use in Europe indicates that the most prevalent substance is cannabis (Observatorio Europeo de las Drogas y las Toxicomanías [EMCDDA], 2016).

Knowing these data, although European crime statistics indicate a decrease (EUROSTAT, 2010), the number of delinquent children in Spain is more than 15000 (INE, 2014) and 59970 citizens are in prison (Secretaría General de Instituciones Penitenciarias, 2016). These worrying figures about early consumption and crime motivated us to research a possible association of risk further. Therefore, the purpose of our systematic review was to know the results of the latest papers that study the influence of the substance use variable on the development of criminal behavior, and therefore confirm if it is really a risk factor in the appearance of crime. Acknowledging the direction of this influence and the synthesis of the results of empirical studies of the last five years could be 
relevant for the development of crime prevention programs and the possible participation of substance use in its etiology.

\section{Method}

\section{Procedure}

The research procedure began on July 11, 2016 at four databases: Scopus, Medline, Psicodoc and PsycINFO. The selection of articles ended on July 27, 2016. The selection followed a hierarchical strategy consisting in selecting the papers after reading the title, abstract and keywords first, to check if they followed the Guidelines marked by the inclusion and exclusion of established criteria. The next step was to read the whole documents and review their quality through the STROBE statement -Strengthening the Reporting of Observational studies in Epidemiology- (Von Elm et al., 2008). Studies which did not present more than two quality criteria were discarded.

The search words used were adapted to the language that each database allowed and the results were screened according to: period of time, language and document type ("articles" and "reviews"). The terms used were:

"predictors OR epidemiology OR "risk factors" AND delinquency OR crime OR "criminal behavior" OR offense OR felony OR crim* OR delinq* AND drugs $O R$ "substance abuse" OR "drug use" OR "substance use" OR alcohol OR cocaine OR cannabis OR heroin OR drug* AND "case control" OR cohort OR prospective OR longitudinal».

Inclusion criteria consists in: observational or quasiexperimental empirical studies published between 2010 and 2015; studies which evaluated causal relationships between the use of drugs, substances or alcohol, and crime and violent or criminal behavior; studies whose design allows the estimation of this factor as a risk or relative risk factor (longitudinal, case-control, retrospective, prospective, or cohort design, odds ratio analysis, relative risk analysis); studies whose designs or data analysis aimed to estimating relative risk, vulnerability or causality, based on the ideas and methods presented by Pita, Vila and Carpente (1997) regarding the estimation of risk; studies which have been published in English, Spanish or Portuguese; studies which have samples of population that have committed crimes or consumed drugs in the present or in the past and finally, studies whose data are both official and self-reported, because some papers such as Dubow, Huesmann, Boxer and Smith (2014) state that there is a moderate to high correspondence between the data collected through self-reports and the official data. We removed descriptive studies, studies related to the concept of recidivism and papers focused on a very specific population.

\section{Results}

The results were taken from twenty studies coming from Scopus, eight from Medline and four from PsycINFO. The information extracted from the studies was organized using a table which details the characteristics of the sample, the type of analysis and the main results of each study.

A $78 \%$ of the empirical studies included in this review confirm that the consumption (or exposure) of some type of substance is a risk factor in the appearance of criminal behavior. From the 32 studies included in this review, $30 \%$ focused on alcohol as a risk factor, $24 \%$ on cannabis and the rest on drugs, other substances and poly-drug use. All but one of the articles referring to the consumption of alcohol find this substance as a risk factor for committing crimes. All papers focusing on cannabis use confirm that it is a risk factor for crime. Most of included studies had a longitudinal design.

\section{Discussion}

Overall, the results of the review articles indicate that severe substance disorder (Barrett Barrett, Teesson, \& Mills, 2014; McKinlay, Corrigan, Horwood, \& Fergusson, 2014; Pullmann, 2010) and poly-drug use increase the risk of criminal involvement (Marel et al., 2013). 
Cannabis represents a risk for control and clinical populations (Brook, Lee, Finch \& Brook, 2014). Specifically, it relates to crimes against property and drug offenses (Green, Doherty, Stuart, \& Ensminger, 2010; Pedersen, \& Skarhamar, 2010). However, Cannabis use is not a risk factor for violent crime because it is associated with a decrease in aggressive behavior (White, Fite, Pardini, Mun, \& Loeber, 2013). Nevertheless, in clinical populations, this is the substance which is most related to violent behavior (Carabellese, Candelli, Martinelli, La Tegola, \& Catanesi, 2013).

Studies that link alcohol consumption with the occurrence of criminal behavior indicate that it represents a risk factor for aggressive behavior (White et al., 2013), violent crime (Dietze et al., 2013; Maldonado-Molina, Reingle, \& Jennings, 2011) and being arrested (Boden, Fergusson, \& Hordwood, 2013; Green, Doherty, Zebrak, \& Esminger, 2011; Jennings, Piquero, Rocque, \& Farrington, 2015; Reingle, Jennings, Lynne-Landsman, Cottler, \& Maldonado-Molina, 2013; Salom et al., 2014; Terranova et al., 2013). Other studies conclude that drinking alcohol and consuming marijuana (Reingle, Jennings, \& Komro, 2013) is not a risk for ending up in jail. However, it should be noticed that not all of those who commit crimes go to prison. In addition, alcohol consumption has been shown to be highly implicated in impulsive offenses (Boden et al., 2013).

On the other hand, some studies indicate that prenatal exposure to cocaine in the first trimester is a risk factor for further development of criminal behavior (Lambert et al., 2013; Richardson, Goldschmidt, Larkby \& Day, 2015). Gerteis et al. (2011) and they also indicate that exposure to cocaine is not a risk factor whereas exposure to tobacco is. Nevertheless, D'Onofrio, Van Hulle, Goodnight, Rathouz and Lahey (2012) with a significantly larger sample, conclude that exposure to tobacco is not a risk factor for criminal behavior when controlling other variables.

In terms of inadequate drug consumption, it is not a predictor of criminal behavior as it is the case with substances such as varenicline and AAS (Lundholm, Frisell, Lichtenstein, \& Langström, 2015; Molero, Lichtenstein, Zetterqvist, Gumpert, \& Fazel, 2015). However, inade- quate consumption of drugs was linked with getting into fights (Drazdowski, Jäggi, Borre, \& Kliewer, 2015; Tucker et al., 2015).

It is important to highlight that most of studies included focus on the consumption of alcohol and marijuana or poly-consume. In the future, it would be interesting to study the influence of the consumption of synthetic drugs on violent or criminal behavior, due to the increase of its current consumption, and its possible implication as a risk factor.

Another important point that must be mentioned is the limitations that this review can present. Firstly, we would like to point out the possible information bias, because some articles not included in the databases consulted may have been left out of the review. On the other hand, the use of self-reports when extracting sample information from some of the included articles may cause these data not to correspond $100 \%$ with reality.

It can be concluded that in the last five years, research has confirmed that the abuse of substances (alcohol and cannabis) and poly-drug use (especially in the adolescent stage) represent a risk for the development of criminal behavior or for the severity of the crime, but not all substances are associated with all types of crime. In addition, the studies that appear here do not find consensus in that there is a reciprocal relationship between consumption and crime.

In short, it is important to note that knowing if the substance use variable represents a real risk factor for the development of criminal behavior, which was the objective of the review, is useful for the development of crime prevention programs and acting in penitentiary centers. In addition, this could not only have the purpose of crime prevention, but also be useful for the promotion of health and quality of life of adolescent and young population. 